\title{
The Role of the Epicardium in the Formation of the Cardiac Valves in the Mouse
}

\author{
Marie M. Lockhart, Maurice van den Hoff, and Andy Wessels
}

\begin{abstract}
In recent years, insights into the role of the epicardium in cardiac development have significantly changed. An important contribution to this increasing knowledge comes from the availability of mouse models that facilitate the study of the fate of the epicardial cell lineage and that allow epicardial-specific manipulation of expression of genes involved in regulation of epicardial cell behavior. In this contribution we will discuss our growing understanding of the role of the epicardium and epicardially derived cells in the formation of the atrioventricular valve leaflets. We will illustrate how epicardially derived cells specifically contribute to the development of the leaflets that derive from the lateral atrioventricular cushions, and we will discuss the role of Bmp signaling, through the $\mathrm{Bmp}$ receptor BmpR1A/Alk3, in the regulation of the preferentially migration of EPDCs into the parietal AV valve leaflets.
\end{abstract}

\section{Keywords}

Epicardium • Valves • Bmp2 • Bmp4 • Alk3

M.M. Lockhart, Ph.D. • A. Wessels, Ph.D. $(\bowtie)$

Department of Regenerative Medicine and Cell Biology, Medical University of South Carolina, 173 Ashley Avenue, Room BSB-648B, P.O. Box 250508, Charleston, SC 29425, USA

e-mail: wesselsa@musc.edu

M. van den Hoff, Ph.D.

Department of Anatomy, Embryology and Physiology, Academic Medical Center, Heart Failure Research Center, Meibergdreef 15, 1105AZ Amsterdam, The Netherlands

T. Nakanishi et al. (eds.), Etiology and Morphogenesis of Congenital Heart Disease,

DOI 10.1007/978-4-431-54628-3_20 


\subsection{Introduction}

\subsubsection{The AV Valves and Their Leaflets}

The atrioventricular (AV) valves are important cardiac components that, when properly formed, prevent the retrograde flow of blood through the AV junction during ventricular systole. The precursor tissues of the AV valves are the endocardial AV cushions, formed by (1) the accumulation of cardiac jelly in the AV canal followed by (2) the population of these cushions by endocardially derived cells (ENDCs) resulting from an endocardial epithelial-to-mesenchymal transformation (or endoEMT) [1]. Not all AV cushions develop at the same time. The two "major" $\mathrm{AV}$ cushions (the inferior and superior cushion) form first, while the two "lateral" cushions form later $[2,3]$. The major AV cushions will eventually fuse together. They will also fuse with the mesenchymal cap of the primary atrial septum and the dorsal mesenchymal protrusion (DMP) to form the AV mesenchymal complex [4]. This process is essential in the partition of the left and right atrial and ventricular components. The AV mesenchymal complex is also involved in the formation of the septal leaflet of the right $\mathrm{AV}$ valve and the aortic (or anterior) leaflet of the left AV valve $[4,5]$. The lateral AV cushions are significantly smaller than the major AV cushions and develop at the lateral myocardial AV junctions. Just like the major AV cushions, the lateral AV cushions become populated with ENDCs. The right lateral cushion eventually forms the parietal leaflet of the right $\mathrm{AV}$ valve, while the left lateral cushion forms the parietal (or mural/posterior) leaflet of the left AV valve (Fig. 20.1).
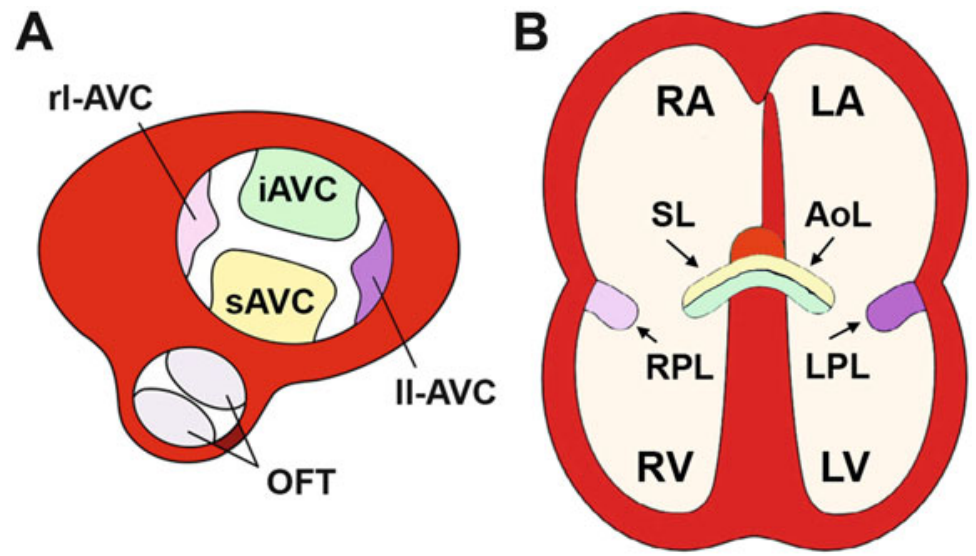

Fig. 20.1 This cartoon shows the fate of the individual AV cushions. The superior and inferior $\mathrm{AV}$ cushion (sAVC and iAVC) fuse at the midline and give rise to the septal leaflet of the right AV valve (SL) and the aortic leaflet (AoL) of the left AV valve. The right lateral AV cushion (rlAVC) forms the right parietal leaflet of the right AV valve (RPL), whereas the left lateral AV cushion forms the left parietal leaflet of the left AV valve (LPL) 


\subsubsection{The Epicardium and Epicardially Derived Cells (EPDCs)}

In the mouse, epicardial development starts around embryonic day 9.5 (ED9.5) when the proepicardium, a heterogeneous cluster of cells, can be seen as a "cauliflower-like" mesothelial structure at the inferior margin of the cardiac sinus venosus at the venous pole of the heart. Around ED10, proepicardial cells reach and attach to the myocardium. Subsequently, they migrate out over the surface of the developing heart, ultimately covering the heart entirely. As a result of an epicardial epithelial-to-mesenchymal transition (epiEMT), epicardially derived cells (EPDC) are generated that migrate into the sub-epicardial space and into the underlying myocardium. Over the last 15 years or so, a series of cell fate studies in avian and murine models have been published reporting that EPDCs can, or have the potential to, differentiate in multiple cell types including coronary smooth muscle cells, interstitial fibroblasts, coronary endothelium, and potentially cardiomyocytes [6-9]. It is important to note that the contribution of EPDCs to the coronary endothelium and myocardium remains controversial.

\subsubsection{The Contribution of EPDCs to the Developing AV Valves}

In recent papers we have discussed the cascade of epicardial-related events involved in the formation and development of the AV junction [7, 10]. This process starts with the establishment of the AV-epicardium. After the formation of the AV-epicardium, subsequent AV-epiEMT leads to the generation of AV-EPDCs, a process regulated by a variety of factors. These AV-EPDCs eventually form the AV sulcus, which can be seen as a mesenchymal wedge between the expanding atrial and ventricular myocardial walls. As AV-EPDCs from the sulcus start to penetrate the AV myocardial junction, the formation of the annulus fibrosus is initiated. The annulus fibrosus is a fibrous tissue plane that physically and electrically separates the atrial from the ventricular working myocardium. From the annulus fibrosus, a subset of AV-EPDCs continues to migrate into the parietal AV valve leaflets, where they eventually comprise a large percentage of the mesenchymal cells found of the leaflet (Fig. 20.2). Just like the ENDCs found in the developing leaflets, the AV-EPDCs in the valves eventually become valve interstitial cells (VICs). It is important to note that very few AV-EPDCs are found in the leaflets that are developing from the fused major AV cushions [7]. 


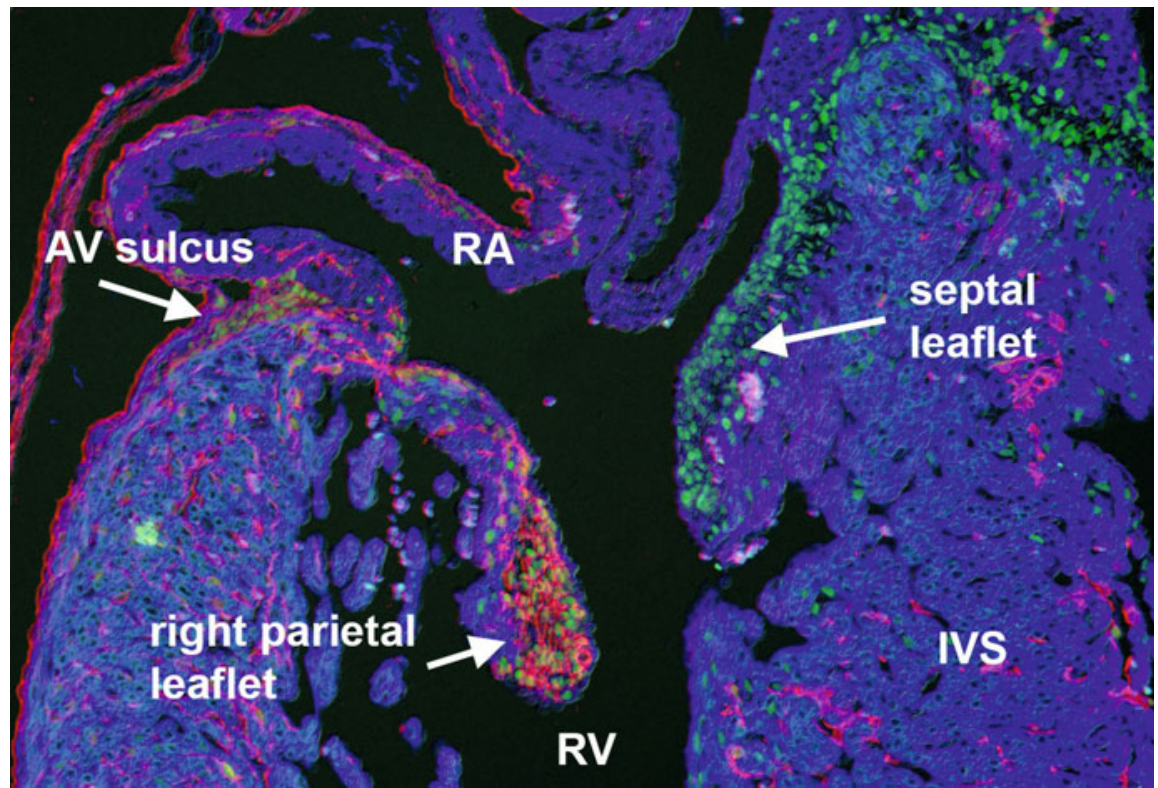

Fig. 20.2 A section of a 17ED Wt1-cre mouse crossed with a ROSA $26^{\mathrm{mT} / \mathrm{mG}}$ reporter mouse was stained for the presence of epicardial and epicardially derived cells ( red), the transcription factor Sox9 (green), and cardiac myosin heavy chain (blue). The section shows the abundant presence of EPDCs in the right parietal leaflets and the virtual absence of EPDCs in the septal leaflet of the right $\mathrm{AV}$ valve

\subsection{The Role of Bmp Signaling in Regulating the Contribution of EPDC to the AV Valves}

\subsubsection{Epicardial-Specific Deletion of the Bmp Receptor BmpR1A/ Alk3 Leads to Disruption of AV Junction Development}

To elucidate the mechanisms that regulate the events that are responsible for the contribution of AV-EPDCs to the tissues of the AV junction, we investigated the importance of bone morphogenetic protein (Bmp) signaling in the epicardium. Bmp isoforms are known to be involved in a variety of developmental steps in heart development. In particular Bmp2, found at high levels in the AV myocardium, has been shown to be important for the development of the AV cushions by promoting endoEMT [11]. In our study, we determined that Bmp4 is highly expressed in the AV-epicardium and in the AV-EPDCs that form the AV sulcus. Furthermore, strong phospho-Smad-1/5/8 labeling of the AV-EPDCs indicates that canonical Bmp signaling is active in these cells, strongly suggesting that this signaling pathway is important in regulating the events associated with the cascade of epicardial-related events at the AV junction [12]. To test the hypothesis that Bmp 


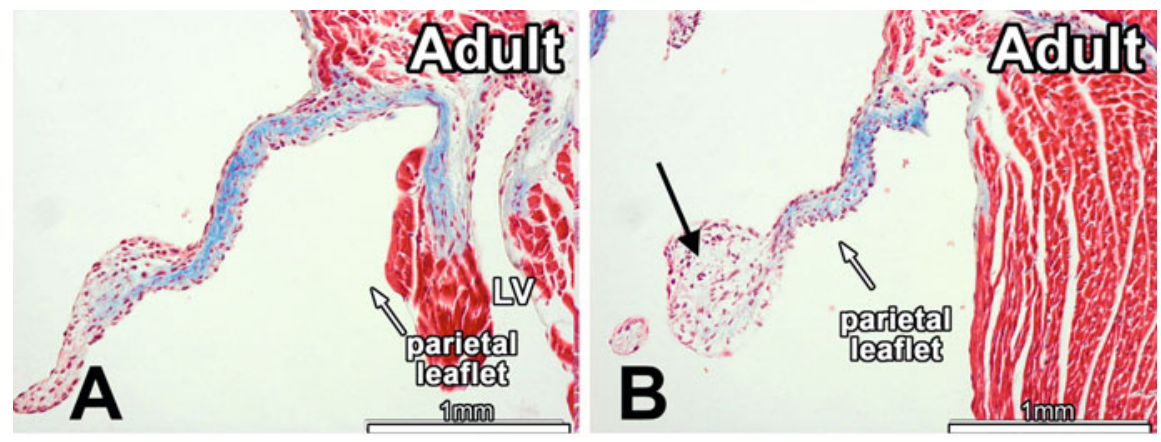

Fig. 20.3 Masson's trichrome staining of adult wild-type (a) and Wt1-cre;Alk3 mouse hearts. Panel (b) demonstrates the myxomatous phenotype of the left parietal leaflet of the epicardial Alk3 knockout mouse (arrow in b)

signaling is important for the development of the structures that rely on the contribution of AV-EPDCs, we deleted the Bmp receptor Alk3 from the epicardial and epicardially derived cells using an epicardial-specific cre-mouse (the Wt1-cre mouse [7, 12]). This approach resulted in a decrease in the size of the AV sulcus, an inhibition of the formation of the annulus fibrosus, and a reduction of the number of EPDCs that migrate into the parietal AV valve leaflets. Remarkably, electrophysiological analysis of postnatal Wt1-cre;Alk3 mice did not show, despite the defect in the formation of the annulus fibrosus, ventricular pre-excitation [12]. In addition, and also quite unexpectedly, postnatal Wt1-Cre;Alk3 showed a myxomatous mitral valve phenotype, particularly of the left parietal leaflet (Fig. 20.3).

\subsubsection{Discussion}

Our studies convincingly show that Bmp signaling plays a critical role in the establishment of the mesenchymal and fibrous tissues at the AV junction. We propose that during normal development, $\mathrm{AV}$-epiEMT is a crucial process as it is responsible for generating the critical amount of AV-EPDCs needed for the subsequent formation of the annulus fibrosus and the associated migration of AV-EPDCs into the parietal leaflets. We believe that perturbation of Bmp signaling by conditionally deleting Alk3 from the epicardial cell population inhibits $\mathrm{AV}$-epiEMT resulting in a domino effect leading to (1) inhibition of AV sulcus formation, (2) failure of the annulus fibrosus to form, and (3) reduction of the number of EPDCs that migrate into the AV valve leaflets (Fig. 20.4).

\subsubsection{Future Direction and Clinical Implications}

Myxomatous valve disease is generally considered an acquired disease, often observed in patients with mitral valve prolapse. The observed myxomatous changes 


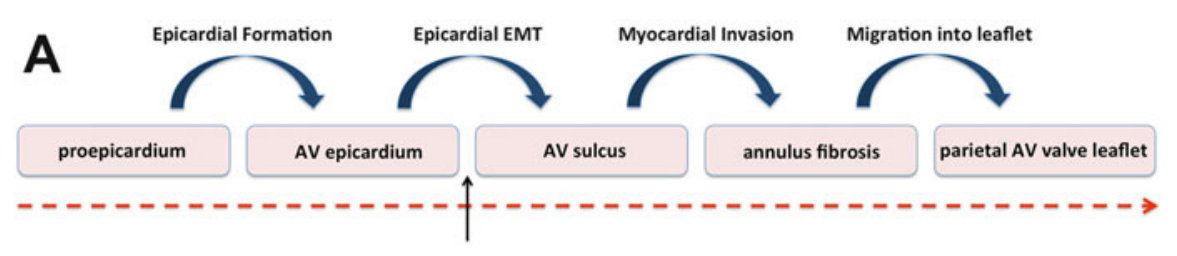

Bmp signaling

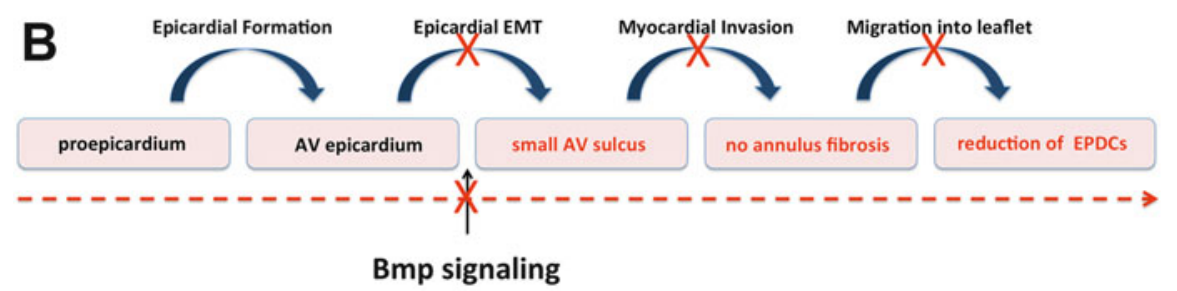

Fig. 20.4 Simplified hypothetical model showing the cascade of epicardial-related events at the $\mathrm{AV}$ junction. (a) In normal development, Bmp signaling positively regulates epiEMT resulting in the formation of the AV sulcus and subsequent development of annulus fibrosus, followed by the migration of EPDCs into the parietal leaflets. (b) When Bmp signaling is perturbed, AV sulcus formation and all AV-epicardial events downstream of this are inhibited

in the leaflets of the postnatal Wt1-cre;Alk3 mice, however, are associated with a reduced influx of EPDCs within those leaflets during embryonic development. We therefore propose that perturbation in the normal development of EPDCs in the AV junction might play the role in the etiology of myxomatous valve degeneration in human heart disease.

Acknowledgments The authors would like to acknowledge the financial support by NIH grants P30 GM103342 (A.W.), UL1RR029882 and UL1TR000062 (A.W.), R01HL033756 (A.W.) American Heart Association Grant-in-Aid 13GRNT16220004 (A.W.), American Heart Association Predoctoral grant 12PRE11340000 (M.M.L.), European Community's Sixth Framework Program Grant LSHM-CT-2005-018630 (MJBvdH), and Netherlands Heart Foundation Grant 1996M002 (MJBvdH).

Open Access This chapter is distributed under the terms of the Creative Commons AttributionNoncommercial 2.5 License (http://creativecommons.org/licenses/by-nc/2.5/) which permits any noncommercial use, distribution, and reproduction in any medium, provided the original author(s) and source are credited.

The images or other third party material in this chapter are included in the work's Creative Commons license, unless indicated otherwise in the credit line; if such material is not included in the work's Creative Commons license and the respective action is not permitted by statutory regulation, users will need to obtain permission from the license holder to duplicate, adapt or reproduce the material. 


\section{References}

1. Markwald RR, Fitzharris TP, Manasek FJ. Structural development of endocardial cushions. Am J Anat. 1977; 148:85-119.

2. Wessels A, Markman MW, Vermeulen JL, Anderson RH, Moorman AF, Lamers WH. The development of the atrioventricular junction in the human heart. Circ Res. 1996;78:110-7.

3. Wessels A, Sedmera D. Developmental anatomy of the heart: a tale of mice and man. Physiol Genomics. 2003;15:165-76.

4. Snarr BS, Wirrig EE, Phelps AL, Trusk TC, Wessels A. A spatiotemporal evaluation of the contribution of the dorsal mesenchymal protrusion to cardiac development. Dev Dyn Off Publ Am Assoc Anat. 2007;236:1287-94.

5. Lamers WH, Viragh S, Wessels A, Moorman AF, Anderson RH. Formation of the tricuspid valve in the human heart. Circulation. 1995;91:111-21.

6. Cai CL, Martin JC, Sun Y, Cui L, Wang L, Ouyang K, Yang L, Bu L, Liang X, Zhang X, et al. A myocardial lineage derives from tbx 18 epicardial cells. Nature. 2008;454:104-8.

7. Wessels A, van den Hoff MJ, Adamo RF, Phelps AL, Lockhart MM, Sauls K, Briggs LE, Norris RA, van Wijk B, Perez-Pomares JM, et al. Epicardially derived fibroblasts preferentially contribute to the parietal leaflets of the atrioventricular valves in the murine heart. Dev Biol. 2012;366:111-24.

8. Zhou B, Ma Q, Rajagopal S, Wu SM, Domian I, Rivera-Feliciano J, Jiang D, von Gise A, Ikeda S, Chien KR, et al. Epicardial progenitors contribute to the cardiomyocyte lineage in the developing heart. Nature. 2008;454:109-13.

9. Zhou B, Pu WT. More than a cover: epicardium as a novel source of cardiac progenitor cells. Regen Med. 2008;3:633-5.

10. Lockhart MM, Phelps AL, van den Hoff MJ, Wessels A. The epicardium and the development of the atrioventricular junction in the murine heart. J Dev Biol. 2014;2:1-17.

11. Ma L, Lu MF, Schwartz RJ, Martin JF. Bmp2 is essential for cardiac cushion epithelialmesenchymal transition and myocardial patterning. Development. 2005;132:5601-11.

12. Lockhart MM, Boukens BJ, Phelps AL, Brown CL, Toomer KA, Burns TA, Mukherjee RD, Norris RA, Trusk TC, van den Hoff MJ et al. Alk3 mediated bmp signaling controls the contribution of epicardially derived cells to the tissues of the atrioventricular junction. Dev Biol. 2014;396:8-18. 